\title{
Emergence and Clonal Spread of CTX-M-65-Producing Escherichia coli From Retail Meat in Portugal
}

\author{
Célia Leão ${ }^{1,2}$, Lurdes Clemente ${ }^{1,3}$, Laura Moura ${ }^{1,4}$, Anne Mette Seyfarth ${ }^{5}$, \\ Inge M. Hansen ${ }^{5}$, Rene S. Hendriksen ${ }^{5}$ and Ana Amaro ${ }^{1 *}$
}

\begin{abstract}
${ }^{1}$ Laboratory of Bacteriology and Mycology, National Institute of Agrarian and Veterinary Research (INIAV, IP), Oeiras, Portugal, ${ }^{2}$ MED - Mediterranean Institute for Agriculture, Environment and Development, Évora, Portugal, ${ }^{3}$ Faculty of Veterinary Science, CIISA- Centre for Interdisciplinary Research in Animal Health, Lisbon, Portugal, ${ }^{4}$ Faculty of Pharmacy, University of Lisbon, Lisbon, Portugal, ${ }^{5}$ EURL-AR, European Reference Laboratory for Antimicrobial Resistance, Technical University of Denmark (DTU), National Food Institute, Lyngby, Denmark
\end{abstract}

OPEN ACCESS

Edited by:

Tarja Sironen,

University of Helsinki, Finland

Reviewed by:

Jian-Hua Liu,

South China Agricultural University,

China

Andres Felipe Opazo-Capurro,

University of Concepcion, Chile

*Correspondence:

Ana Amaro

ana.amaro@iniav.pt

Specialty section:

This article was submitted to Antimicrobials, Resistance

and Chemotherapy,

a section of the journal

Frontiers in Microbiology

Received: 14 January 2021 Accepted: 10 May 2021

Published: 20 July 2021

Citation:

Leão C, Clemente L, Moura L, Seyfarth AM, Hansen IM, Hendriksen RS and Amaro A (2021)

Emergence and Clonal Spread of CTX-M-65-Producing Escherichia coli From Retail Meat in Portugal.

Front. Microbiol. 12:653595.

doi: 10.3389/fmicb.2021.653595
The emergence and dissemination of resistance to third- and fourth-generation cephalosporins among Enterobacteriaceae from different sources impose a global public health threat. Here, we characterized by whole-genome sequencing four Escherichia coli strains harboring the bla $\mathrm{CTX}_{-\mathrm{M}-65}$ gene identified among 49 isolates from beef and pork collected at retail. The genomic content was determined using the Center for Genomic Epidemiology web tools. Additionally, the prediction and reconstruction of plasmids were conducted, the genetic platform of the blacTX-M-65 genes was investigated, and phylogenetic analysis was carried out using 17 other genomes with the same sequence type and harboring the bla $\mathrm{CTX}-\mathrm{M}-65$ gene. All strains harbored bla $\mathrm{CTX}_{\mathrm{M}-\mathrm{M}-65}$, bla OXA-1, and blaTEM-1B, and one also carried the bla $\mathrm{SHV}_{\mathrm{SH}} 12$ gene. Other resistance genes, namely, gnrS2, aac(6')-lb-c, dfrA14, sul2, tet $A$, and $m p h A$, were present in all the genomes; the $m c r-1.1$ gene was identified in the colistinresistant strains. They belong to sequence type 2179, phylogenetic group B1, and serotype 09:H9 and carried plasmids Incl, IncFIC(FII), and IncFIB. All strains share an identical genetic environment with IS903 and ISECP1 flanking the blacTX-M-65 gene. It seems likely that the blactX-M-65 gene is located in the chromosome in all isolates based on deep in silico analysis. Our findings showed that the strains are clonally related and belong to two sub-lineages. This study reports the emergence of CTX-M-65producing $E$. coli in Portugal in food products of animal origin. The chromosomal location of the bla $\mathrm{CTX}-\mathrm{M}-65$ gene may ensure a stable spread of resistance in the absence of selective pressure.

Keywords: Escherichia coli, retail meat, ESBL, WGS, CTX-M-65, chromosome

\section{INTRODUCTION}

Bacteria harboring antimicrobial resistance genes can be spread to humans, constituting a global public health concern (O’Neill, 2016). Of particular importance is the resistance to $\beta$-lactam antibiotics, enzymatic inactivation being the most common mechanism of resistance through which $\beta$-lactamases cause the cleavage of the $\beta$-lactam ring (Blair et al., 2015). Extended-spectrum $\beta$-lactamase (ESBL) is a group of enzymes that hydrolyze oxymino-beta-lactam antibiotics, 
conferring resistance to a wide variety of $\beta$-lactams, including penicillins, first-, second-, third-, and fourth-generation cephalosporins, and monobactams (Bush, 2018). These enzymes can be inhibited by $\beta$-lactam inhibitors such as clavulanic acid, sulbactam, and tazobactam through the covalent link to the serine residue active site (Tooke et al., 2019). Mobile genetic elements, particularly plasmids, are involved in the spread of ESBL genes, resulting in the rapid increase of ESBL-producing bacteria among different sources (Rozwandowicz et al., 2018; Silva et al., 2019). ESBLs are classified into several enzymatic groups, of which CTX-M, OXA, SHV, and TEM are frequently observed, CTX-M being the most prevalent (Silva et al., 2019; Palmeira and Ferreira, 2020). Currently, 230 CTX-M variants have been described according to GenBank records (last accessed on January 4, 2021). In Europe, the most frequently reported variants of Enterobacteriaceae species isolated from foodproducing animals and food products are CTX-M-1, CTX-M-14, CTX-M-15, and CTX-M-2, CTX-M-15 being associated with outbreaks of severe extraintestinal infections in humans caused by the multidrug-resistant (MDR) Escherichia coli ST131 (Silva et al., 2019; Palmeira and Ferreira, 2020). In Portugal, the CTX-M-1, CTX-M-14, and CTX-M-32 variants were reported as the most prevalent in commensal and pathogenic E. coli isolated from food-producing animals and food products (Clemente et al., 2019; Silva et al., 2019).

In 2008, a new variant of the CTX-M family identified as CTX-M-65, belonging to the CTX-M-9 group and cluster 14, was described for the first time in E. coli isolated from a human urine sample in the United States (Doi et al., 2008). CTX-M-65 differs from CTX-M-14 by two amino acid substitutions, namely, alanine by valine at position $77(\mathrm{~A} 77 \mathrm{~V})$ and serine by arginine at position 274 (S274R). In 2017, Tate and colleagues reported the emergence of a CTX-M-65 Salmonella Infantis isolated from food animals, retail chickens, and humans in the United States that is highly resistant to most of the studied $\beta$-lactam antimicrobials (Tate et al., 2017). In Europe, the CTX-M-65 variant has also been reported from Italy in Salmonella Infantis isolated from broilers and humans (Franco et al., 2015) and from the Netherlands in E. coli from cattle (Palmeira and Ferreira, 2020). Globally, the enzyme is widely distributed, with reports from China, Korea, and South America in E. coli isolated from humans, foodproducing animals, retail chickens, and in a giant anteater from a zoo (Zheng et al., 2012; Bartoloni et al., 2013; Rao et al., 2014; Tate et al., 2017; Furlan et al., 2019; Park et al., 2019; VinuezaBurgos et al., 2019; Wang et al., 2020). It is noteworthy to mention that a multidrug carbapenemase strain of Klebsiella pneumoniae co-producing CTX-M-65 was the causative agent of a severe nosocomial outbreak in China (Zhan et al., 2017).

In the present study, four CTX-M-65-producing E. coli isolated from beef and pork samples collected at retail in 2017 were characterized by whole-genome sequencing (WGS). Here, we described the molecular epidemiology of the resistance genes, the identification of the plasmids, and the bla $a_{\mathrm{CTX}}-\mathrm{M}-65$ genetic environment. Moreover, we determined and described the genetic relatedness with other $E$. coli genomes for better insights into the public health impact of an ESBL producer rarely found in Europe.

\section{MATERIALS AND METHODS}

\section{Bacterial Isolates and Antimicrobial Susceptibility Testing}

Two hundred and twenty beef and 220 pork samples were collected at retail stores across mainland Portugal in compliance with the European Commission Implementing Decision of November 12, 2013 to monitor and report antimicrobial resistance in zoonotic and commensal bacteria (Commission Decision 652/2013) in 2017. The isolation and identification of extended-spectrum $\beta$-lactamase/plasmid-mediated AmpC $(\mathrm{ESBL} / \mathrm{PMA} \beta)$ E. coli producers from meat samples were performed according to the laboratory protocols defined by the European Union Reference Laboratory for antimicrobial resistance (EURL-AR) ${ }^{1}$. Briefly, $25 \mathrm{~g}$ of each meat sample was mixed with $225 \mathrm{ml}$ of buffered peptone water, followed by incubation at $37^{\circ} \mathrm{C}$ for $18-22 \mathrm{~h}$. Enriched samples were plated onto MacConkey agar supplemented with $1 \mathrm{mg} / \mathrm{L}$ of cefotaxime (Glentham, Corsham, United Kingdom) and incubated at $44^{\circ} \mathrm{C}$ for 18-22 h. Presumptive E. coli colonies were selected for biochemical identification on ChromID ${ }^{\circledR}$ coli agar (bioMérieux, Marcy-l'Étoile, France), and after confirmation, the isolates were sub-cultured and stored at $-80^{\circ} \mathrm{C}$ before further analyses.

The isolates were tested for antimicrobial susceptibility through the determination of the minimum inhibitory concentrations (MICs) using commercially available 96well microplates, EUVSEC and EUVSEC2 (Sensititre ${ }^{\circledR}$, Trek Diagnostic Systems, East Grinstead, United Kingdom) panels, and the results were interpreted according to EUCAST epidemiological breakpoints ${ }^{2}$.

\section{Molecular Characterization of $\beta$-Lactam Resistance}

Resistance mechanisms associated with ESBL/PMA $\beta$ enzymes were screened by PCR using primers targeting bla $a_{\mathrm{TEM}}, b l a_{\mathrm{SHV}}$, $b l a_{\mathrm{OX}}, b l a_{\mathrm{CTX}-\mathrm{M}}, b l a_{\mathrm{ACC}}, b l a_{\mathrm{FOX}}, b l a_{\mathrm{MOX}}, b l a_{\mathrm{DHA}}, b l a_{\mathrm{CIT}}$, and $b l a_{\mathrm{EBC}}$ (Dallenne et al., 2010). Amplified products were purified with ExoSAP-IT ${ }^{\mathrm{TM}}$ (Applied Biosystems ${ }^{\mathrm{TM}}$, Warrington, United Kingdom), followed by Sanger sequencing using the BigDye $^{\circledR}$ Terminator v3.1 Cycle Sequencing Kit (Applied Biosystems). The sequencing of fragments was performed in an automatic sequencer ABI3100 (Applied Biosystems), and the identification of resistance genes was determined using the Basic Local Alignment Search Tool (BLAST) from the NCBI website (Altschul et al., 1990) and The Comprehensive Antibiotic Resistance Database (CARD) (Alcock et al., 2019).

\section{Whole-Genome Sequencing and Bioinformatics Analysis of the CTX-M-65 E. coli Producers}

Four isolates identified as positive for $b l a_{\mathrm{CTX}}-\mathrm{M}-65$ by PCR and Sanger sequencing were further characterized by whole-genome sequencing. The isolates were recovered from pork and beef

\footnotetext{
${ }^{1}$ https://www.eurl-ar.eu/protocols.aspx

${ }^{2}$ https://mic.eucast.org/Eucast2/
} 
samples, collected on the same day from the same retail store in the north of Portugal (INIAV_ECX027 and INIAV_ECX036) and on different days from different retail stores in Lisbon and Tejo Valley (INIAV_ECX016 and INIAV_ECX035).

The genome of two isolates (INIAV_016ECX and INIAV_027ECX) was sequenced at EURL-AR, DTU, Lyngby, Denmark, under the scope of the European Food Safety Authority (EFSA) confirmatory testing. Genomic DNA was extracted using an Invitrogen Easy-DNA $\mathrm{Kit}^{\mathrm{TM}}$ (Invitrogen, Carlsbad, CA, United States) and the DNA concentrations determined using the Qubit dsDNA BR assay kit (Invitrogen). Genomic DNA was prepared for Illumina pair-end sequencing using the Illumina (Illumina, Inc., San Diego, CA, United States) Nextera XT ${ }^{\circledR}$ Guide following the protocol revision C1. A sample of the pooled Nextera XT Libraries was loaded onto an Illumina MiSeq reagent cartridge using MiSeq Reagent Kit v3. The libraries were sequenced using an Illumina MiSeq platform (Illumina). The raw reads were de novo assembled using the assembler pipeline (version 1.4) available from the Center for Genomic Epidemiology $(\mathrm{CGE})^{3}$. Raw sequence data from these two isolates were submitted to the European Nucleotide Archive (ENA) ${ }^{4}$ under study accession numbers: ERS3535656 and ERS3535669.

DNA extraction of the remaining two isolates (INIAV_035ECX and INIAV_036ECX) was carried out at INIAV, Oeiras, Portugal, using the PureLink ${ }^{\circledR}$ Genomic DNA kit (Invitrogen) according to the manufacturer's instructions, with minor modifications. Briefly, the incubation period at $55^{\circ} \mathrm{C}$ was performed for $90 \mathrm{~min}$ and the DNA eluted with $50 \mu \mathrm{l}$ of Tris-HCl buffer, $\mathrm{pH}$ 8.5. The DNA quality and quantity were assessed using a spectrophotometer [NanoDrop ${ }^{\circledR}$ 2000, Thermo Scientific, emergency use authorization (EUA), Waltham, MA, United States] and sequenced using the Illumina HiSeq sequencing technology (NovaSeq 6000 S2 PE150 XP sequencing mode, Eurofins Genomics Europe Sequencing $\mathrm{GmbH}$, Ebersberg, Germany). The raw sequence data from these two isolates were submitted to the ENA under study accession numbers: ERS5493675 and ERS5493676. Raw data quality was assessed by FastQC ${ }^{5}$. BBDuk from the BBTools package ${ }^{6}$ was used to remove possible contamination by adapter sequences and for trimming/removing low-quality reads, all performed with a minimum quality of Q20 using the Phred algorithm, with a minimum read length of 50 and with a $k$-mer length parameter of 19. All pre-processed reads were assembled with SPAdes 3.12.0 (Nurk et al., 2013).

The assembly stats of all the sequenced isolates were calculated using QUAST-5.0.2 (Mikheenko et al., 2018). Contigs with sizes lower than $500 \mathrm{bp}$ were removed, and bioinformatics analysis using tools available at the CGE was performed. The acquired antimicrobial resistance genes and chromosomal point mutations, plasmid replicons, multilocus sequence type (MLST), serotype, fum $\mathrm{C}$ and fim $\mathrm{H}$ type, identification of virulence genes, and pathogenicity were assessed using ResFinder

\footnotetext{
${ }^{3}$ http://cge.cbs.dtu.dk/services/all.php

${ }^{4}$ http://www.ebi.ac.uk/ena

${ }^{5}$ http://www.bioinformatics.babraham.ac.uk/projects/fastqc

${ }^{6}$ http://jgi.doe.gov/data-and-tools/bb-tools/
}

version 4.1 (80\% threshold for $\%$ ID/60\% minimum length) (Zankari et al., 2012; Bortolaia et al., 2020), PlasmidFinder version 2.1 (80\% threshold for\%ID) (Carattoli et al., 2014), MLST version 2.0 (Larsen et al., 2012), SerotypeFinder version 2.0 (85\% threshold for\%ID/60\% minimum length) (Joensen et al., 2015), CHTyper version 1.0 (95\% threshold for\%ID) (Camacho et al., 2009), VirulenceFinder version 2.0 (90\% threshold for\%ID/60\% minimum length) (Joensen et al., 2014), and PathogenFinder version 1.1 (Cosentino et al., 2013), respectively. The phylogenetic group was predicted using the ClermonTyping web-based tool (Beghain et al., 2018; Clermont et al., 2019).

Additionally, PLACNETw (Plasmid Constellation Network) was used to predict and reconstruct the plasmids (Vielva et al., 2017) in specifically assembled contigs of the bacterial genomes. For identifying the genetic platform of the CTX-M-65 enzyme, the contigs containing the bla $a_{\mathrm{CTX}}-\mathrm{M}-65$ gene were annotated using Prokka version 1.14.6 (Seemann, 2014), followed by analysis with Artemis (Carver et al., 2012) and EasyFig version 2.2.5 (Sullivan et al., 2011). A plasmid database, PLSDB, was also used to search for the plasmid nucleotide sequences contained in each of the selected contigs using the "mash screen" option (Galata et al., 2018).

A phylogenetic analysis based on the single nucleotide polymorphisms (SNPs) present in the genomes using CSI Phylogeny version 1.4 (10 reads of minimal depth at SNP positions, $10 \%$ minimal relative depth at SNP positions, $10 \mathrm{bp}$ of minimal distance between SNPs, minimal SNP quality of 30, minimal read mapping quality of 25 , and a minimal $Z$-score of 1.96) (Kaas et al., 2014) from the CGE website was conducted with the E. coli isolates from this study and 17 E. coli genomes with the same sequence type (ST2179) and harboring the bla $a_{\mathrm{CTX}-\mathrm{M}-65}$ gene, retrieved from EnteroBase (Zhou et al., 2020). The tree was visualized using FigTree version v1.4.37.

\section{RESULTS}

\section{Characterization of Antimicrobial Resistance}

From 49 isolates of E. coli phenotypically resistant to thirdgeneration cephalosporins, a total of 10 different bla genes were identified in $42 \mathrm{ESBL}$ and seven PMA $\beta$ producers: bla $a_{\mathrm{CTX}-\mathrm{M}-1}(n=8), b l a_{\mathrm{CTX}-\mathrm{M}-15}(n=5), b l a_{\mathrm{CTX}-\mathrm{M}-27}(n=2)$, bla $a_{\mathrm{CTX}-\mathrm{M}-55}(n=1), b l a_{\mathrm{CTX}-\mathrm{M}-9}(n=1), b l a_{\mathrm{CTX}-\mathrm{M}-14}(n=7)$, bla $_{\mathrm{CTX}}-\mathrm{M}-32(n=9)$, bla $_{\mathrm{CTX}}-\mathrm{M}-65(n=4)$, bla $_{\mathrm{SHV}-12}(n=5)$, and bla $\mathrm{CMY}-2(n=7)$.

The bla $a_{\mathrm{CTX}-\mathrm{M}-65}$ gene was detected in four isolates $(8.2 \%)$ recovered from beef $(3 / 26)$ and pork $(1 / 23)$ retail meat samples, and all exhibited a MDR phenotype, being resistant to ciprofloxacin, nalidixic acid, azithromycin, chloramphenicol, tetracycline, trimethoprim, and sulfamethoxazole. Resistance to colistin was observed in all, except isolate INIAV_ECX035 (Table 1).

\footnotetext{
${ }^{7}$ http://tree.bio.ed.ac.uk/software/figtree/
} 
TABLE 1 | Results of the minimum inhibitory concentrations ( $\mu \mathrm{g} / \mathrm{ml})$ obtained by antimicrobial susceptibility testing.

\begin{tabular}{|c|c|c|c|c|c|}
\hline Antibiotic & Breakpoints & INIAV_ECX016 & INIAV_ECX027 & INIAV_ECX035 & INIAV_ECX036 \\
\hline Ampicillin & 8 & $>64$ & $>64$ & $>64$ & $>64$ \\
\hline Cefepime & 0.125 & 4 & 2 & 4 & 8 \\
\hline Cefotaxime & 0.25 & 64 & 64 & 64 & $>64$ \\
\hline Cefoxitin & 8 & 8 & 8 & 8 & 8 \\
\hline Ceftazidime & 0.5 & 1 & 1 & 32 & 2 \\
\hline Ciprofloxacin & 0.064 & $>8$ & $>8$ & $>8$ & $>8$ \\
\hline Nalidixic acid & 16 & $>128$ & $>128$ & $>128$ & $>128$ \\
\hline Colistin & 2 & 4 & 4 & $\leq 1$ & 4 \\
\hline Ertapenem & 0.064 & $\leq 0.015$ & $\leq 0.015$ & $\leq 0.015$ & $\leq 0.015$ \\
\hline Imipenem & 0.5 & 0.25 & 0.25 & 0.25 & 0.5 \\
\hline Meropenem & 0.125 & $\leq 0.03$ & $\leq 0.03$ & $\leq 0.03$ & $\leq 0.03$ \\
\hline Tetracycline & 8 & 64 & $>64$ & 64 & $>64$ \\
\hline Sulfamethoxazole & 64 & $>1,024$ & $>1,024$ & $>1,024$ & $>1,024$ \\
\hline Trimethoprim & 2 & $>32$ & $>32$ & $>32$ & $>32$ \\
\hline Chloramphenicol & 16 & 128 & 128 & 128 & 128 \\
\hline Gentamicin & 2 & 1 & 1 & 1 & 2 \\
\hline Azithromycin & 16 & 32 & 32 & 64 & 64 \\
\hline Tigecycline & 1 & 0.5 & 0.5 & 0.5 & 0.5 \\
\hline Temocillin & 32 & 8 & 8 & 8 & 8 \\
\hline Cefotaxime/clavulanic acid & 0.25 & $\leq 0.06$ & $\leq 0.06$ & 0.12 & 0.12 \\
\hline Ceftazidime/clavulanic acid & 0.5 & $\leq 0.12$ & 0.25 & $\leq 0.12$ & 0.25 \\
\hline
\end{tabular}

\section{Genome Analysis of the CTX-M-65 E. coli Producers}

The assemblies of the reads originated between 70 and 120 contigs and a genome size of about $5 \mathrm{~Gb}$. The genotypic traits of the four isolates, their resistome and mobilome, are summarized in Table 2. According to the ResFinder tool, three $\beta$-lactam-encoding genes were found in all isolates, namely, $b l a_{\mathrm{CTX}-\mathrm{M}-65}, b l a_{\mathrm{OXA}-1}$, and $b l a_{\mathrm{TEM}-1 \mathrm{~B}}$. Moreover, one isolate (INIAV_ECX035) also carried bla $a_{\mathrm{SHV}-12}$. The $m c r$ - 1.1 gene was identified in the three isolates resistant to colistin, and plasmidmediated quinolone resistance (PMQR) genes, namely, qnrS2 and $a a c\left(6^{\prime}\right)-I b-c r$, were detected in all isolates. Additional genes conferring resistance to trimethoprim/sulfamethoxazole ( $d f r A 14$ and sul2), tetracycline (tetA), and azithromycin ( $m p h A)$ were also present. Point mutations were identified at the gyrA and parC subunits of DNA, namely, serine by lysine at position 83 (S83L) and serine by isoleucine at position 80 (S80I), respectively, conferring resistance to quinolones. Based on these results, the phenotype and genotype were in accordance.

According to the PlasmidFinder tool, IncFIC (FII) and IncFIB replicons were identified in all isolates, and p0111 was also predicted in one isolate (INIAV_ECX016). Isolate INIAV_ECX035 also harbored the IncI1-I plasmid, while the remaining isolates carried the IncI2 plasmid. All strains have identical profiles regarding MLST (ST2179), fumC65/fim $\mathrm{H} 32$ alleles, serotype (O9:H9), and phylogroup (B1). The somatic antigen of the INIAV_ECX036 isolate was not typable. VirulenceFinder predicted the presence of five virulence factors (lpfA, iroN, iss, cma, and gad) in all isolates except one (INIAV_ECX016), which carries four as gad is absent.
Using PLACNETw, a new assembly was generated from the raw reads, and a network was produced representing the contigs defined as belonging to the chromosome and plasmids, according to the reference genomes from the software database. The contigs were identified as belonging to plasmids based on the recognition of relaxase and/or replicon protein sequences. A manual pruning of the original network (Figure 1A) was performed according to Lanza et al. (2014) to reconstruct the graphical representation of the genome (Figure 1B). With the FASTA files obtained from this assembly, a new analysis with PlasmidFinder and ResFinder was performed to identify in which contigs were the antimicrobial resistance genes and plasmids located. Hereafter, it was possible to pinpoint the exact position of the resistance genes in the genome's graphical representation.

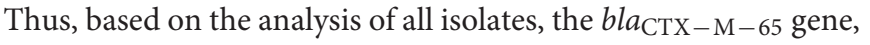
along with floR and $m d f \mathrm{~A}$, was identified in contigs belonging to the chromosome, and the remaining resistance genes, including the other bla genes, were located in contigs belonging to plasmids (Figure 1B). In isolates INIAV_ECX016, INIAV_ECX027, and INIAV_ECX036, genes bla $a_{\mathrm{CTX}-\mathrm{M}-65}$ and floR were located in the same contig, and $m d f \mathrm{~A}$ was in a different contig, while in isolate INIAV035, the three genes were in different contigs. Most resistance genes were located in IncFIC (FII) and IncFIB plasmids, except bla $a_{\mathrm{SHV}-12}$ and $m c r 1.1$, which were carried in IncI- 1 and IncI2, respectively.

The analysis of the genetic platform of the $b l a_{\mathrm{CTX}}-\mathrm{M}-65$ genes using the EasyFig tool revealed that all isolates have the IS903 (IS5 family) and ISEcp1 (IS1380 family) flanking the bla $a_{\mathrm{CTX}-\mathrm{M}-65}$ gene, and all but one isolate also harbored the transposon TnAs3 (Tn3 family), IS1006 (IS6 family) and ISVsa3 (IS91 family). In this isolate (INIAV_ECX035), the contig starts 
TABLE 2 | Genomic characterization of the four CTX-M-65-producing E. coli isolates by whole-genome sequencing.

\begin{tabular}{|c|c|c|c|c|c|}
\hline \multicolumn{2}{|l|}{ Features } & \multirow{2}{*}{$\begin{array}{l}\text { INIAV_ECX016 } \\
\text { bla } \\
\text { bla } \text { TEM-M-1B }_{\text {TEM }}, \text { bla } \\
\text { OXA-1 }\end{array}$} & \multirow{2}{*}{$\begin{array}{l}\text { INIAV_ECX027 } \\
\text { bla } 2 \text { CTX-M-65, } \\
\text { bla } \\
\text { TEM-1B, bla OXA-1 }\end{array}$} & \multirow{2}{*}{$\begin{array}{l}\text { INIAV_ECX035 } \\
\text { bla } \\
\text { blatX-M-65, } \\
\text { bla } \text { blaM-1B }_{\mathrm{SHV}-12} \text { bla }\end{array}$} & \multirow{2}{*}{$\begin{array}{l}\text { INIAV_ECX036 } \\
\text { bla } 1 \text { CTX-M-65, } \\
\text { bla } \\
\text { TEM-1B }, \text { bla OXA-1 }\end{array}$} \\
\hline $\begin{array}{l}\text { Antibiotic resistance } \\
\text { determinants }\end{array}$ & $\begin{array}{l}\text { Ampicillin, Cefepime, } \\
\text { Cefotaxime, Cefoxitin, } \\
\text { Ceftazidime }\end{array}$ & & & & \\
\hline & $\begin{array}{l}\text { Ciprofloxacin, Nalidixic } \\
\text { acid }\end{array}$ & $\begin{array}{l}\text { QnrS2, aac }\left(6^{\prime}\right) l b-c r \\
\text { gyrA S83L, parC S80I }\end{array}$ & $\begin{array}{l}\text { QnrS2, aac }\left(6^{\prime}\right) l b-c r \\
\text { gyrA S83L, parC S80I }\end{array}$ & $\begin{array}{l}\text { QnrS2, aac }\left(6^{\prime}\right) / b-c r \\
\text { gyrA S83L, parC S80I }\end{array}$ & $\begin{array}{l}\text { QnrS2, aac }\left(6^{\prime}\right) l b-c r \\
\text { gyrA S83L, parC S80I }\end{array}$ \\
\hline & Colistin & $m c r-1.1$ & $m c r-1.1$ & - & $m c r-1.1$ \\
\hline & Tetracycline & $\operatorname{tet}(A)$ & $\operatorname{tet}(A)$ & $\operatorname{tet}(A)$ & $\operatorname{tet}(A)$ \\
\hline & Sulphamethoxazole & sul2 & sul2 & sul2, sul1 & sul2 \\
\hline & Trimethoprim & dfrA14 & dfrA14 & dfrA17 & dfrA14 \\
\hline & Chloramphenicol & catB3, floR & catB3, floR & catB3, floR & catB3, floR \\
\hline & Azithromycin & $m p h(A)$ & $m p h(A)$ & $m p h(A)$ & $m p h(A)$ \\
\hline & Rifampicin & ARR-3 & ARR-3 & ARR-3 & ARR-3 \\
\hline & Aminoglycoside & $a p h\left(3^{\prime \prime}\right)-I b, a p h(6)-l d$ & $a p h\left(3^{\prime \prime}\right)-I b, a p h(6)-/ d$ & $\begin{array}{l}\operatorname{aad} A 5, \operatorname{aph}\left(3^{\prime \prime}\right)-1 b, \\
\operatorname{aph}(6)-l d\end{array}$ & $a p h\left(3^{\prime \prime}\right)-I b, a p h(6)-l d$ \\
\hline & Other & $m d f(A)$ & $m d f(A)$ & $\operatorname{mdf}(A)$ & $m d f(A)$ \\
\hline \multicolumn{2}{|l|}{ Plasmid replicons } & $\begin{array}{l}\text { Incl2, plncFIB, p0111, } \\
\text { IncFIC }\end{array}$ & Incl2, plncFIB, IncFIC & $\begin{array}{l}\text { Incl1-I, IncFIB, IncFIC } \\
\text { (FII) }\end{array}$ & Incl2, IncFIB, IncFIC(FII) \\
\hline \multicolumn{2}{|l|}{ MLST } & ST2179 & ST2179 & ST2179 & ST2179 \\
\hline \multicolumn{2}{|l|}{ Serotype } & O9:H9 & O9:H9 & $\mathrm{O9:H9}$ & $\mathrm{H} 9$ \\
\hline \multicolumn{2}{|l|}{ Virulence genes } & IpfA, iroN, iss, cma & IpfA, iroN, iss, cma, gad & IpfA, iroN, iss, cma, gad & IpfA, iroN, iss, cma, gad \\
\hline \multicolumn{2}{|l|}{ fumC/fimH type } & fumC65/fimH32 & fumC65/fimH32 & fumC65/fimH32 & fumC65/fimH32 \\
\hline \multicolumn{2}{|l|}{ Pathogenicity } & Yes (93.6\%) & Yes (93.6\%) & Yes (93.3\%) & Yes (93.6\%) \\
\hline \multicolumn{2}{|l|}{ Phylogenetic group } & B1 & B1 & B1 & $\mathrm{B} 1$ \\
\hline \multicolumn{2}{|l|}{ Sample source } & Bovine & Swine & Bovine & Bovine \\
\hline \multicolumn{2}{|l|}{ No. of contigs } & 90 & 77 & 74 & 79 \\
\hline \multicolumn{2}{|c|}{ Total length of genome (bp) } & $5,115,923$ & $5,012,767$ & $5,015,983$ & $5,058,472$ \\
\hline \multicolumn{2}{|c|}{ N50 (bp) } & 140,276 & 142,205 & 282,032 & 232,144 \\
\hline
\end{tabular}
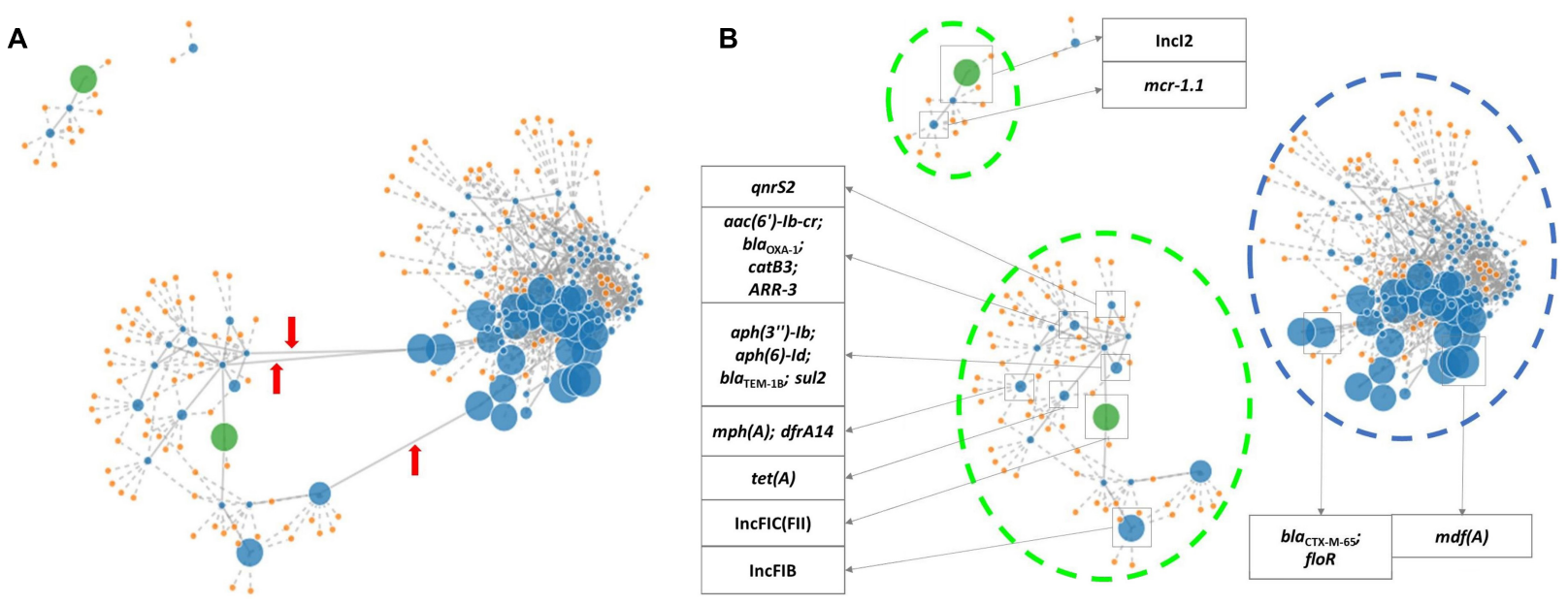

FIGURE 1 | PLACNETw (Plasmid Constellation Network) representation of the INIAV_ECX036 genome. (A) The original network. Red arrows indicate the contig linkages that were pruned. (B) The pruned network, where contigs containing the antimicrobial resistance genes and plasmids are identified according to ResFinder and PlasmidFinder. Blue dots indicate contigs (the dot size is proportional to the size of the contig); orange dots are the reference genomes from the tool database; green dots are contigs with relaxase and replicon protein of the plasmid; blue broken lines indicate contigs identified as chromosome; and green broken lines are contigs identified as plasmids.

in a different position, and its genetic platform seems to be not fully represented (Figure 2A). Consequently, the genetic platform is split and the downstream elements (TnAs3, IS1006, and ISVsa3) are not represented in this contig, being in a different one. Looking at a larger region of the contigs (Figure 2B), it is possible to realize that all four contigs have high homology between each other. The alignment of each contig using EasyFig with the plasmid sequences identified by PlasmidFinder revealed 


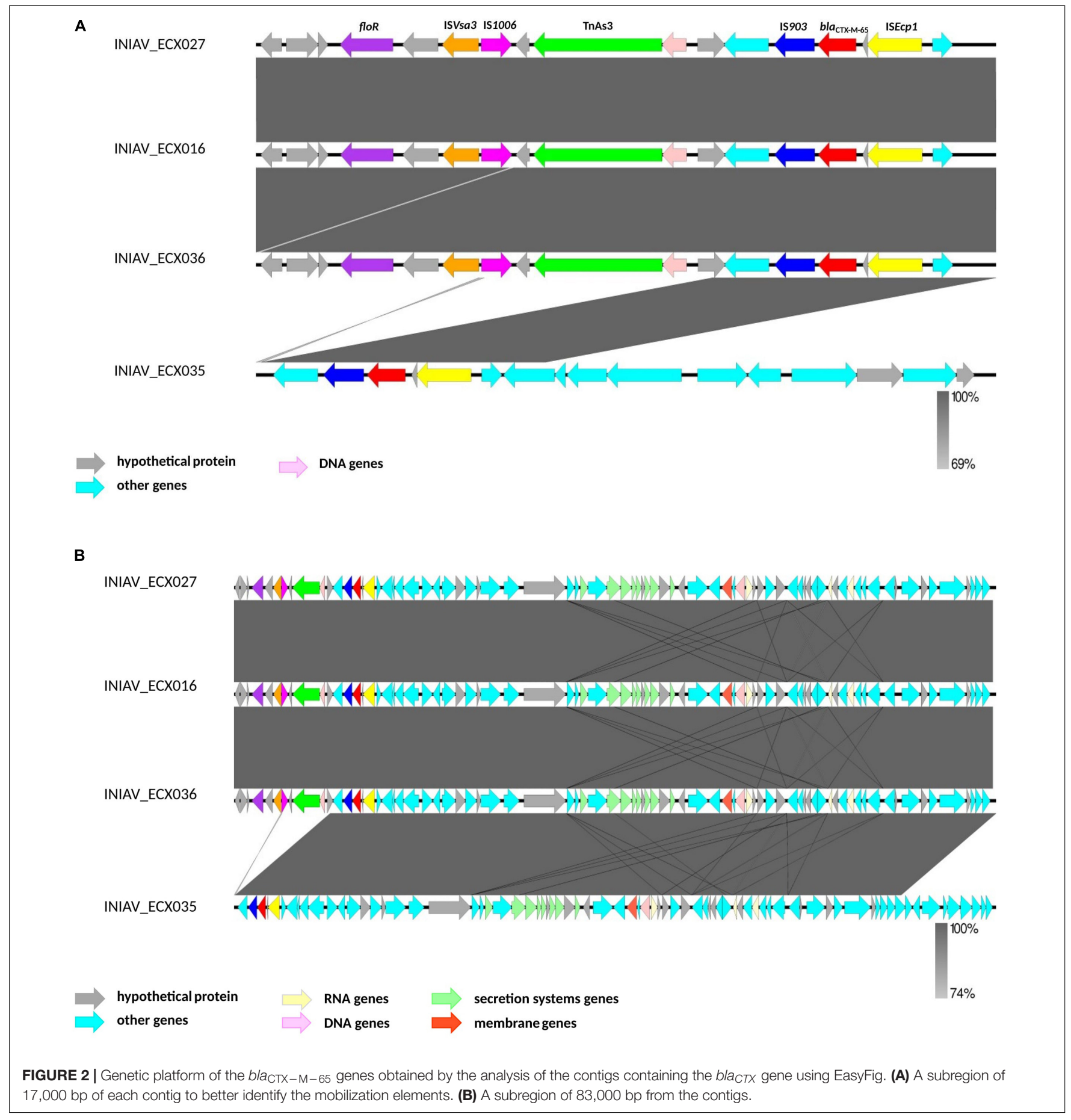

no homology between those sequences. Comparison of the sizes of the contigs containing the bla $a_{\mathrm{CTX}-\mathrm{M}-65}$ gene (ranging from 227,681 to $340,574 \mathrm{bp}$, except for the INIAV_ECX035 isolate with 82,631 bp in length) with the sizes of the plasmids identified in this study (ranging from 64,015 to $99,159 \mathrm{bp}$ ) revealed that the contigs are mostly wider than the plasmids. Furthermore, no plasmids were also identified, using the "mash screen" option of the PLSDB tool, in the contig's input sequence containing the bla $a_{\mathrm{CTX}-\mathrm{M}-65}$ gene from each isolate.
An unrooted phylogenetic tree (radial cladogram option) using E. coli ATCC 25922 as the reference genome was constructed using 21 genomes from different geographic regions worldwide and isolated from multiple sources (Figure 3). Multiple phylogenetic groups can be distinguished in the tree: one group includes two strains, one from Nigeria and one from Colombia isolated from poultry and human samples; another phylogenetic group including European strains from the United Kingdom, Hungary, and Italy, isolated mainly 


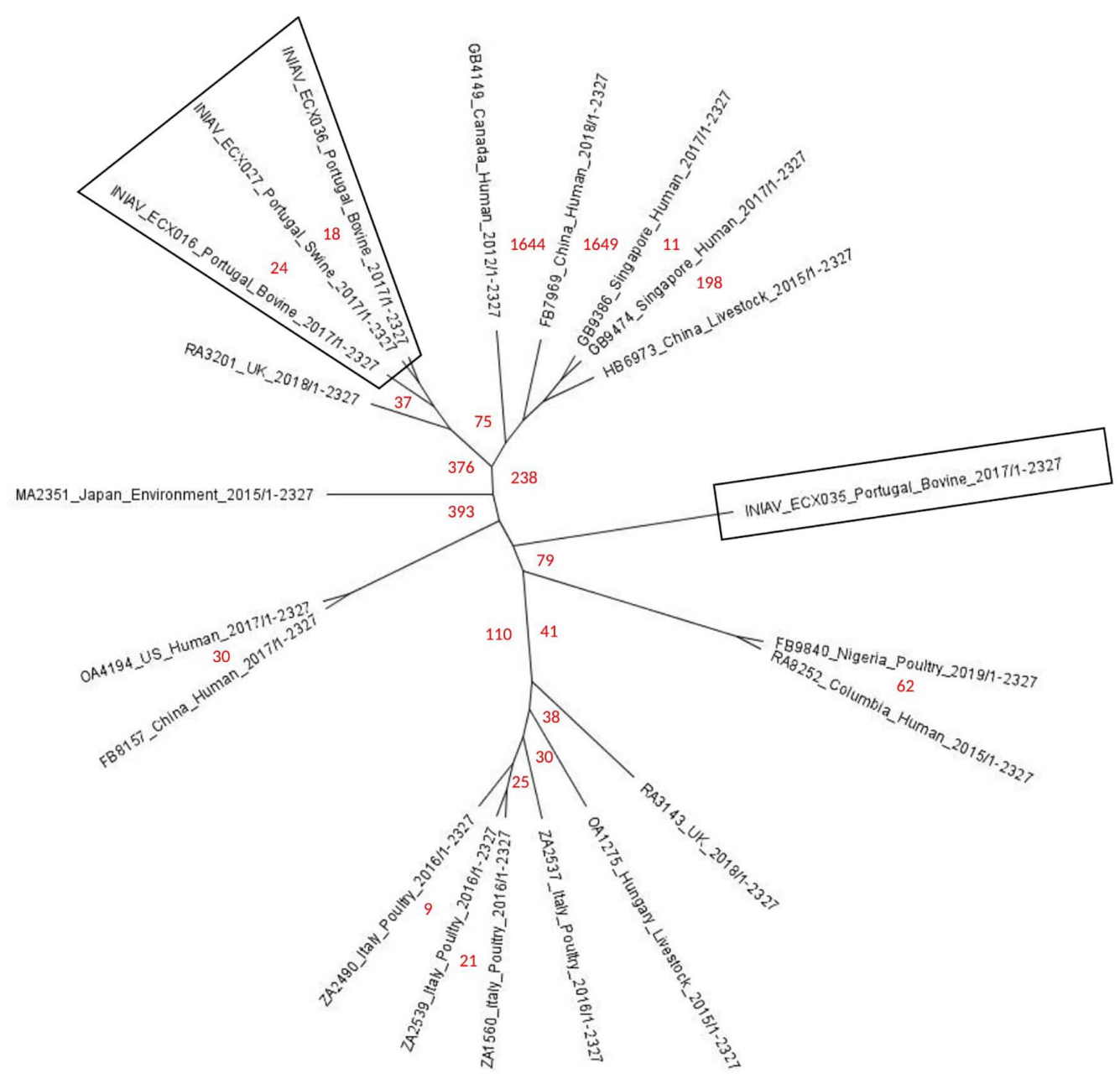

FIGURE 3 | Phylogenetic tree of the 21 ST2179 Escherichia coli strains harboring the bla $\mathrm{CTX}_{-\mathrm{M}-65}$ gene generated by single nucleotide polymorphism (SNP) analysis using the CSIPhylogeny tool and visualized with FigTree (unrooted radial cladogram options). Numbers in red represent the number of SNPs between the strains from distinct geographic regions and sources. Portuguese strains are inside boxes.

from poultry; other groups formed with one strain from the United States and one strain from China isolated from human samples; another group includes strains from China, Singapore, and Canada isolated from human and livestock samples; and two groups with only one strain each from Portugal and Japan isolated from bovine and environmental samples. All shared about 43,430 SNPs against the reference genome. The SNP analysis showed FB7969_China_Human_2018 as the strain having more SNPs, between 1,636 and 1,746. Most shared less than 112 SNPs with the four strains from this study, except for HB6973_China_Livestock_2015 and MA2351_Japan_Environment_2015 separated by between 219 and 394 SNPs. Three Portuguese strains were grouped with one strain from the UK (RA3201_UK_2018), separated by 37 and 45 SNPs. The fourth Portuguese strain (INIAV_ECX035) is separated from the remaining three from this study by 85 and 101 SNPs, being in a different phylogenetic group. The most closely related strains from this study are INIAV_ECX016 and INIAV_ECX036, sharing only 8 SNPs between each other; these two strains were obtained from beef samples collected from distinct geographic regions in Portugal.

\section{DISCUSSION}

In this study, four CTX-M-65-producing E. coli were identified among $49 \mathrm{ESBL} / \mathrm{PMA} \beta$ producers isolated from meat. Here, bla $a_{\mathrm{CTX}-\mathrm{M}-65}$ is identified in Portugal for the first time in food of animal origin, with no reports linking to human infections; a complete characterization by WGS of the four MDR E. coli harboring this gene is described. E. coli harboring the bla $a_{\mathrm{CTX}}-\mathrm{M}-65$ gene is commonly found in food-producing animals and meat from Southwest Asian and South American countries (Rao et al., 2014; Na et al., 2019; Vinueza-Burgos et al., 2019; Zurita et al., 2019). Although rarely occurring in Europe, previous studies have reported CTX-M-65-producing E. coli from Dutch beef calves (Ceccarelli et al., 2019) and wild birds from Switzerland (Zurfluh et al., 2019). In our study, three isolates were from beef and one from pork; therefore, we 
cannot confirm the source of the CTX-M-65 E. coli detected in meat. There are several potential sources of bacteria in meat, including the animals from which the meat was derived, cross-contamination from other products, equipment and the environment, and the workers who are producing and handling the meat (EFSA and ECDC, 2019).

Based on PLACNETw, the bla $a_{\mathrm{CTX}}-\mathrm{M}-65$ genes were located in contigs with homology to the chromosome. Moreover, the contigs containing this gene are wider than the plasmids identified in this study, and none of the four contigs showed homology to any sequence of the identified plasmids, reinforcing that the bla $\mathrm{CTX}-\mathrm{M}-65$ gene is in the chromosome. The chromosomal location of $b l a_{\mathrm{CTX}}-\mathrm{M}$ genes has already been reported, namely, bla $\mathrm{CTX}-\mathrm{M}-2$ (Zhao and $\mathrm{Hu}, 2013$; Ferreira et al., 2014), bla $a_{\mathrm{CTX}-\mathrm{M}-14}$ and bla $a_{\mathrm{CTX}-\mathrm{M}-15}$ (Hamamoto

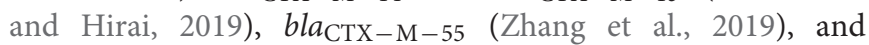
bla $\mathrm{CTX}-\mathrm{M}-65$ (He et al., 2017).

The successful spread of ESBL enzymes is based on their ability to disseminate their resistance genes on mobile genetic elements to other bacteria, the skill to expand their spectrum of activity, and the acquisition of point mutations (Bush, 2018; Galal et al., 2018; Tooke et al., 2019). Nevertheless, the chromosomal location of the resistance genes can benefit the stable propagation of resistance, regardless of the bacterial host's habitat (Yoon et al., 2020). The genetic platform analysis revealed that all share an identical genetic environment with IS903 and ISEcp1 flanking the bla $a_{\mathrm{CTX}}-\mathrm{M}-65$ gene downstream and upstream, respectively. ISEcp1 is one of the most important insertion sequences associated with bla $a_{\mathrm{CTX}-\mathrm{M}}$ genes (Canton et al., 2012; Zhao and $\mathrm{Hu}, 2013$ ) and $\mathrm{Tn} 3$, a conjugative transposon. These mobile genetic elements found in the chromosome may promote excision and reintegration in a new chromosome or transference to other bacteria through a conjugative plasmid (Canton et al., 2012).

Particularly worrying is the co-occurrence of genes encoding resistance to other critically important antimicrobials, namely, fluoroquinolones, macrolides, and polymyxins, except for isolate INIAV_ECX035, which was susceptible to colistin. This isolate also carried the bla $a_{\mathrm{SH}-12}$ gene, exhibiting a higher MIC to ceftazidime ( $\mathrm{MIC}=32 \mu \mathrm{g} / \mathrm{ml}$ ) compared to the remaining isolates $(\mathrm{MIC}=1-2 \mu \mathrm{g} / \mathrm{ml}$ ), which is in accordance with the previously described (Maina et al., 2011).

Of note is that all isolates were resistant to azithromycin and harbored the $m p h(A)$ gene, confirming this gene's relevant role in macrolide susceptibility. As previously reported (Gomes et al., 2019), most of the $m p h(A)$-carrying isolates show a $\mathrm{MIC}>32 \mu \mathrm{g} / \mathrm{L}$, as observed in our study, where the MIC values were between 32 and $64 \mu \mathrm{g} / \mathrm{L}$. Moreover, other antimicrobial resistance genetic determinants were found in the isolates, including those also associated with resistance to antimicrobials frequently used in the rearing of food-producing animals, such as sulfamethoxazole (sul1 and sul2), trimethoprim ( $d f r A 14$ and dfrA17), phenicols (catB3 and floR), tetracycline (tet $A)$, and aminoglycosides [aph( $\left.3^{\prime \prime}\right)$-Ib and $a p h(6)$-Id] (ESVAC, 2020).

In all isolates, different replicon-typing plasmids were identified [IncFIC(FII) and IncFIB] carrying most of the resistance genes. IncF plasmids are frequently described from human and animal sources and are considered epidemic resistance plasmids, bearing the greatest variety of resistance genes in Enterobacteriaceae (Rozwandowicz et al., 2018). Although the precise gene location on plasmids was not determined in this study, based on PLACNETw, mor-1.1 was located on IncI2 replicon-typing plasmid. IncI2 plasmids have been associated with the mobilization of $\mathrm{mcr}$ genes widely spread in Europe in E. coli isolates from animals and humans (Rozwandowicz et al., 2018; Migura-Garcia et al., 2020). The IncI-1 plasmid predominantly described in Europe was identified in the INIAV_ECX035 strain carrying bla $a_{\mathrm{SHV}-12}$, in accordance with previous reports (Rozwandowicz et al., 2018).

WGS analysis also revealed that all isolates belong to ST2179, phylogroup B1, and fumC65/fimH32 type, suggesting that all have a common clonal origin, with minor differences. Recently, E. coli ST2179 bearing the bla $a_{\mathrm{CTX}-\mathrm{M}-65}$ gene but belonging to phylogroup A was reported from ducks in South Korea ( $\mathrm{Na}$ et al., 2019). Isolates belonging to phylogroup B1 are commonly associated with non-pathogenic commensal E. coli reported from humans, animals, and food products (Bailey et al., 2010; Coura et al., 2015; Scheinberg et al., 2017; Belaynehe et al., 2018; Zurita et al., 2019).

The phylogenetic analysis revealed three isolates (INIAV_ECX016, INIAV_ECX027, and INIAV_ECX036) grouped in the same cluster, showing high genetic homology between each other. The UK strain (RA3201_UK_2018) was closely related to the Portuguese strains with 37-45 SNPs. The in silico analysis of the UK strain revealed the bla $a_{\mathrm{CTX}-\mathrm{M}-65}$ gene to also be located in the chromosome. These findings suggest the clonal spread of CTX-M-65-producing E. coli isolates in Europe. INIAV_ECX035 is separated from INIAV_ECX027 by 101 SNPs and from the remaining two strains by 85 SNPs. Although from different animal species, the isolates from the same retail store (INIAV_ECX027 and INIAV_ECX036) are closely related, pointing out the hypothesis of cross-contamination. Moreover, the phylogenetic tree and the WGS analysis suggest that the four isolates belong to two sub-lineages, one composed of the three strains that grouped and the second with the fourth strain. INIAV_ECX035 showed some differences regarding the resistance genes and plasmids compared with the other three strains: the presence of the bla $a_{\mathrm{SHV}-12}$ gene conferring a higher resistance to ceftazidime and the absence of resistance to colistin, also the presence of the IncI1-I plasmid instead of the IncI2 plasmid carrying the $m c r-1.1$ gene. These genetic differences may justify the higher number of SNPs found and the existence of two evolutionary sub-lineages suggesting that the CTX-M-65 variant is emerging in our country.

The emergence and clonal spread of E. coli harboring

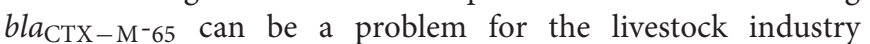
and human health, given their multidrug resistance profile to critically important antimicrobials and their presence in the food supply chain. To our knowledge, this is the first time that CTX-M-65 is identified in Portugal in food products of animal origin. The chromosomal addition of the bla $a_{\mathrm{CTX}-\mathrm{M}-65}$ gene may ensure the spread of resistance in the absence of selective pressure. A better understanding of the factors that contribute to the emergence and dissemination of ESBL genes rarely seen 
in Europe, but highly prevalent in Southwest Asian and South American countries, is strongly advisable and is worthy of close monitoring. Tourism and migration flow to Portugal and the trade treaties established between countries by importing meat and meat products may be sources of cross-contamination with uncommon MDR strains, facilitating their dissemination to the community.

\section{DATA AVAILABILITY STATEMENT}

The datasets presented in this study can be found in online repositories. The names of the repository/repositories and accession number(s) can be found below. https://www.ebi.ac.uk/ ERS3535656, ERS3535669, ERS5493675, and ERS5493676.

\section{AUTHOR CONTRIBUTIONS}

CL contributed to the whole-genome sequencing (WGS) experiments, bioinformatics analysis, and interpretation of the data and wrote the manuscript. LM contributed to the

\section{REFERENCES}

Alcock, B. P., Raphenya, A. R., Lau, T. T. Y., Tsang, K. K., Bouchard, M., Edalatmand, A., et al. (2019). CARD 2020: antibiotic Resistome Surveillance with the Comprehensive Antibiotic Resistance Database. Nucleic Acids Res. 48, D517-D525. doi: 10.1093/nar/gkz935

Altschul, S. F., Gish, W., Miller, W., Myers, E. W., and Lipman, D. J. (1990). Basic local alignment search tool. J. Mol. Biol. 215, 403-410. doi: 10.1016/S00222836(05)80360-2

Bailey, J., Pinyon, J., Anantham, S., and Hall, R. (2010). Commensal Escherichia coli of healthy humans: a reservoir for antibiotic-resistance determinants. J. Med. Microbiol. 59, 1331-1339. doi: 10.1099/jmm.0.022475-0

Bartoloni, A., Pallecchi, L., Riccobono, E., Mantella, A., Magnelli, D., Di Maggio, T., et al. (2013). Relentless increase of resistance to fluoroquinolones and expanded-spectrum cephalosporins in Escherichia coli: 20 years of surveillance in resource-limited settings from Latin America. Clin. Microbiol. Infect. 19, 356-361. doi: 10.1111/j.1469-0691.2012.03807.x

Beghain, J., Bridier-Nahmias, A., Le Nagard, H., Denamur, E., and Clermont, O. (2018). Clermontyping: an easy-to-use and accurate in silico method for Escherichia genus strain phylotyping. Microb. Genom. 4:e000192. doi: 10.1099/ mgen.0.000192

Belaynehe, K., Won Shin, S., and Sang Yoo, H. (2018). Interrelationship between tetracycline resistance determinants, phylogenetic group affiliation and carriage of class 1 integrons in commensal Escherichia coli isolates from cattle farms. BMC Vet. Res. 14:340. doi: 10.1186/s12917-018-1661-3

Blair, J. M., Webber, M. A., Baylay, A. J., Ogbolu, D. O., and Piddock, L. J. (2015). Molecular mechanisms of antibiotic resistance. Nat. Rev. Microbiol. 13, 42-51. doi: $10.1038 /$ nrmicro3380

Bortolaia, V., Kaas, R. F., Ruppe, E., Roberts, M. C., Schwarz, S., Cattoir, V., et al. (2020). ResFinder 4.0 for predictions of phenotypes from genotypes. J. Antimicrob. Chemother. 75, 3491-3500. doi: 10.1093/jac/dkaa345

Bush, K. (2018). Past and present perspectives on $\beta$-lactamases. Antimicrob. Agents Chemother. 62, e01076-18. doi: 10.1128/AAC.01076-18

Camacho, C., Coulouris, G., Avagyan, V., Ma, N., Papadopoulos, J., Bealer, K., et al. (2009). BLAST+: architecture and applications. BMC Bioinformatics 10:421. doi: 10.1186/1471-2105-10-421

Canton, R., González-Alba, J. M., and Galán, J. C. (2012). CTX-M enzymes: origin and diffusion. Front. Microbiol. 3:110. doi: 10.3389/fmicb.2012.00110

Carattoli, A., Zankari, E., Garcia-Fernandez, A., Voldby, L. M., Lund, O., Villa, L., et al. (2014). PlasmidFinder and pMLST: in silico detection and typing of laboratory experiments. AS, IH, and RH contributed to the WGS and bioinformatics analysis. LC and AA designed the study, interpreted the data, and reviewed and edited the manuscript. All authors read and approved the manuscript.

\section{FUNDING}

This work was supported by the project PTDC/CVTCVT/28469/2017 "CIAinVET-Food-producing animals as reservoirs of resistance to Critically Important Antibiotics" financed by the "Fundação para a Ciência e Tecnologia" (FCT), Portugal. CL was also funded by National Funds through FCTFoundation for Science and Technology under the Project UIDB/05183/2020.

\section{ACKNOWLEDGMENTS}

We are grateful to all staff involved in the sampling and laboratory work for their technical support.

plasmids. Antimicrob. Agents Chemother. 58, 3895-3903. doi: 10.1128/AAC. 02412-14

Carver, T., Harris, S. R., Berriman, M., Parkhill, J., and McQuillan, J. A. (2012). Artemis: an integrated platform for visualization and analysis of highthroughput sequence-based experimental data. Bioinformatics 28, 464-469. doi: 10.1093/bioinformatics/btr703

Ceccarelli, D., Kant, A., van Essen-Zandbergen, A., Dierikx, C., Hordijk, J., Wit, B., et al. (2019). Diversity of Plasmids and Genes Encoding Resistance to Extended Spectrum Cephalosporins in Commensal Escherichia coli From Dutch Livestock in 2007-2017. Front. Microbiol. 4:76. doi: 10.3389/fmicb.2019.00076

Clemente, L., Manageiro, V., Correia, I., Amaro, A., Albuquerque, T., Themudo, P., et al. (2019). Revealing mcr-1-positive ESBL-producing Escherichia coli strains among Enterobacteriaceae from food-producing animals (bovine, swine and poultry) and meat (bovine and swine), Portugal, 2010-2015. Int. J. Food Microbiol. 296, 37-42. doi: 10.1016/j.ijfoodmicro.2019.02.006

Clermont, O., Dixit, O. V. A., Vangchhia, B., Condamine, B., Dion, S., BridierNahmias, A., et al. (2019). Characterization and rapid identification of phylogroup G in Escherichia coli, a lineage with high virulence and antibiotic resistance potential. Environ. Microbiol. 21, 3107-3117. doi: 10.11111/14622920.14713

Cosentino, S., Larsen, M. V., Aarestrup, F. M., and Lund, O. (2013). PathogenFinder - Distinguishing Friend from Foe Using Bacterial Whole Genome Sequence Data. PLoS One 8:e77302. doi: 10.1371/journal.pone. 0077302

Coura, F., Diniz, S., Silva, M., Mussi, J., Barbosa, S., Lage, A., et al. (2015). Phylogenetic Group Determination of Escherichia coli Isolated from Animals Samples. ScientificWorldJournal 2015:258424. doi: 10.1155/2015/258424

Dallenne, C., Da Costa, A., Decré, D., Favier, C., and Arlet, G. (2010). Development of a set of multiplex PCR assays for the detection of genes encoding important $\beta$-lactamases in Enterobacteriaceae. J. Antimicrob. Chemother. 65, 490-495. doi: 10.1093/jac/dkp498

Doi, Y., Adams-Haduch, J. M., and Paterson, D. L. (2008). Escherichia coli isolate coproducing 16S rRNA Methylase and CTX-M-type extended-spectrum betalactamase isolated from an outpatient in the United States. Antimicrob. Agents Chemother. 52, 1204-1205. doi: 10.1128/AAC.01320-07

EFSA, and ECDC. (2019). The European Union summary report on antimicrobial resistance in zoonotic and indicator bacteria from humans, animals and food in 2017. EFSA J. 17:e05598. doi: 10.2903/j.efsa.2019.5598

ESVAC. (2020). 10th ESVAC Report Sales of veterinary antimicrobial agents in 31 European countries in 2018. Amsterdam: European Medical Agency. 
Ferreira, J. C., Penha Filho, R. A. C., Andrade, L. N., Berchieri, A., and Darini, A. L. C. (2014). Detection of chromosomal blaCTX-M-2 in diverse Escherichia coli isolates from healthy broiler chickens. Clin. Microbiol. Infect. 20, O623O626. doi: 10.1111/1469-0691.12531

Franco, A., Leekitcharoenphon, P., Feltrin, F., Alba, P., Cordaro, G., Iurescia, M., et al. (2015). Emergence of a Clonal Lineage of Multidrug-Resistant ESBLProducing Salmonella Infantis Transmitted from Broilers and Broiler Meat to Humans in Italy between 2011 and 2014. PLoS One 10:e0144802. doi: 10.1371/ journal.pone.0144802

Furlan, J. P. R., Moura, Q., Gonzalez, I. H. L., Ramos, P. L., Lincopan, N., and Stehling, E. G. (2019). Draft genome sequence of a multidrug-resistant CTX-M65-producing Escherichia coli ST156 colonizing a giant anteater (Myrmecophaga tridactyla) in a Zoo. J. Glob. Antimicrob. Resist. 17, 19-20. doi: 10.1016/j.jgar. 2019.03.005

Galal, L., Abdel Aziz, N. A., and Hassan, W. M. (2018). Defining the relationship between phenotypic and genotypic resistance profiles of multidrug-resistant Enterobacterial clinical isolates. Adv. Exp. Med. Biol. 1214, 9-21. doi: 10.1007/ 5584_2018_208

Galata, V., Fehlmann, T., Backes, C., and Keller, A. (2018). PLSDB: a resource of complete bacterial plasmids. Nucleic Acids Res. 47, D195-D202. doi: 10.1093/ nar/gky1050

Gomes, C., Ruiz-Roldán, L., Mateu, J., Ochoa, T., and Ruiz, J. (2019). Azithromycin resistance levels and mechanisms in Escherichia coli. Sci. Rep. 9:6089. doi: 10. 1038/s41598-019-42423-3

Hamamoto, K., and Hirai, I. (2019). Characterization of chromosomally-located blaCTX-M and its surrounding sequence in CTX-M type extended-spectrum $\beta$-lactamase-producing Escherichia coli isolates. J. Glob. Antimicrob. Resist. 17, 53-57. doi: 10.1016/j.jgar.2018.11.006

He, D., Liu, L., Guo, B., Wu, S., Chen, X., Wang, J., et al. (2017). Chromosomal location of the fosA 3 and blaCTX-M genes in Proteus mirabilis and clonal spread of Escherichia coli ST117 carrying fosA3-positive IncHI2/ST3 or F2:A:B- plasmids in a chicken farm. Int. J. Antimicrob. Agents 49, 443-448. doi: 10.1016/j.ijantimicag.2016.12.009

Joensen, K. G., Scheutz, F., Lund, O., Hasman, H., Kaas, R. S., Nielsen, E. M., et al. (2014). Real-time whole-genome sequencing for routine typing, surveillance, and outbreak detection of verotoxigenic Escherichia coli. J. Clin. Micobiol. 52, 1501-1510. doi: 10.1128/JCM.03617-13

Joensen, K. G., Tetzschner, A. M., Iguchi, A., Aarestrup, F. M., and Scheutz, F. (2015). Rapid and easy in silico serotyping of Escherichia coli using whole genome sequencing (WGS) data. J. Clin. Microbiol. 53, 2410-2426. doi: 10.1128/ JCM.00008-15

Kaas, R. S., Leekitcharoenphon, P., Aarestrup, F. M., and Lund, O. (2014). Solving the Problem of Comparing Whole Bacterial Genomes across Different Sequencing Platforms. PLoS One 9:e104984. doi: 10.1371/journal.pone.0104984

Lanza, V. F., Toro, M., Garcillán-Barcia, M. P., Mora, A., Blanco, J., Coque, T. M., et al. (2014). Plasmid Flux in Escherichia coli ST131 Sublineages, Analyzed by Plasmid Constellation Network (PLACNET), a New Method for Plasmid Reconstruction from Whole Genome Sequences. PLoS Genet. 10:e1004766 doi: 10.1371/journal.pgen.1004766

Larsen, M. V., Cosentino, S., Rasmussen, S., Friis, C., Hasman, H., Marvig, R. L., et al. (2012). Multilocus Sequence Typing of Total Genome Sequenced Bacteria. J. Clin. Micobiol. 50, 1355-1361. doi: 10.1128/jcm.06094-11

Maina, D., Revathi, G., Kariuki, S., and Ozwara, H. (2011). Genotypes and cephalosporin susceptibility in extended-spectrum $\beta$-lactamase producing Enterobacteriaceae in the community. J. Infect. Dev. Ctries. 6, 470-477. doi: $10.3855 /$ jidc. 1456

Migura-Garcia, M., González-López, J., Martinez-Urtaza, M., Aguirre Sánchez, J., Moreno-Mingorance, A., Perez de Rozas, A., et al. (2020). mcr-Colistin Resistance Genes Mobilized by IncX4, IncHI2, and IncI2 Plasmids in Escherichia coli of Pigs and White Stork in Spain. Front. Microbiol. 17:3072. doi: $10.3389 /$ fmicb. 2019.03072

Mikheenko, A., Prjibelski, A., Saveliev, V., Antipov, D., and Gurevich, A. (2018). Versatile genome assembly evaluation with QUAST-LG. Bioinformatics 34, i142-i150. doi: 10.1093/bioinformatics/bty266

Na, S., Moon, D., Choi, M., Oh, S., Jung, D., Sung, E., et al. (2019). Antimicrobial Resistance and Molecular Characterization of ExtendedSpectrum $\beta$-Lactamase-Producing Escherichia coli Isolated from Ducks in South Korea. Foodborne Pathog. Dis. 16, 799-806. doi: 10.1089/fpd.2019.2644
Nurk, S., Bankevich, A., Antipov, D., Gurevich, A., Korobeynikov, A., Lapidus, A., et al. (2013). "Assembling genomes and mini-metagenomes from highly chimeric reads," in Research in computational molecular biology, eds M. Deng, R. Jiang, F. Sun, and X. Zhang (Berlin: Springer), 158-170. doi: 10.1007/978-3642-37195-0 13

O'Neill, J. (2016). Review on Antimicrobial Resistance. Tackling Drug-Resistant Infections Globally: Final report and Recommendations. U.K.: Government of United Kingdom.

Palmeira, J. D., and Ferreira, H. M. N. (2020). Extended-spectrum beta-lactamase (ESBL)-producing Enterobacteriaceae in cattle production - a threat around the world. Heliyon 6:e03206. doi: 10.1016/j.heliyon.2020.e03206

Park, H., Kim, J., Ryu, S., and Jeon, B. (2019). The predominance of blaCTX-M65 and blaCTX-M-55 in extended-spectrum $\beta$-lactamase-producing Escherichia coli from retail raw chicken in South korea. J. Glob. Antimicrob. Resist. 17, 216-220. doi: 10.1016/j.jgar.2019.01.005

Rao, L., Luchao, L., Zeng, Z., Chen, S., He, D., Chen, X., et al. (2014). Increasing prevalence of extended-spectrum cephalosporin-resistant Escherichia coli in food animals and the diversity of CTX-M genotypes during 2003-2012. Vet. Microbiol. 172, 534-541. doi: 10.1016/j.vetmic.2014.06.013

Rozwandowicz, M., Brouwer, M., Fischer, J., Wagenaar, J., Gonzalez-Zorn, B., Mevius, D., et al. (2018). Plasmids carrying antimicrobial resistance genes in Enterobacteriaceae. J. Antimicrob. Chemother. 73, 1121-1137. doi: 10.1093/jac/ $\mathrm{dkx} 488$

Scheinberg, J., Dudley, E., Campbell, J., Roberts, B., Dimarzio, M., Debroy, C., et al. (2017). Prevalence and Phylogenetic Characterization of Escherichia coli and Hygiene Indicator Bacteria Isolated from Leafy Green Produce, Beef, and Pork Obtained from Farmers' Markets in Pennsylvania. J. Food Prot. 80, 237-244. doi: 10.4315/0362-028X.JFP-16-282

Seemann, T. (2014). Prokka: rapid prokaryotic genome annotation. Bioinformatics 30, 2068-2069. doi: 10.1093/bioinformatics/btu153

Silva, N., Carvalho, I., Currie, C., Sousa, M., Igrejas, G., and Poeta, P. (2019). "Extended-Spectrum- $\beta$-Lactamase and Carbapenemase-Producing Enterobacteriaceae in Food-Producing Animals in Europe," in Antibiotic Drug Resistance, Chapter 12, eds J. L. Capelo-Martínez and G. Igrejas (New Jerssey: John Wiley \& Sons, Inc), 261-273. doi: 10.1002/9781119282549.ch12

Sullivan, M. J., Petty, N. K., and Beatson, S. A. (2011). Easyfig: a genome comparison visualizer. Bioinformatics. 27, 1009-1010. doi: 10.1093/bioinformatics/btr039

Tate, H., Folster, J. P., Hsu, C.-H., Chen, J., Hoffmann, M., Li, C., et al. (2017). Comparative analysis of extended-spectrum- $\beta$-lactamase CTX-M-65producing Salmonella enterica serovar Infantis isolates from humans, food animals, and retail chickens in the United States. Antimicrob. Agents Chemother. 61, e00488-17. doi: 10.1128/AAC.00488-17

Tooke, C. L., Hinchliffe, P., Bragginton, E. C., Colenso, C. K., Hirvonen, V. H. A., Takebayashi, Y., et al. (2019). B-Lactamases and B-Lactamase Inhibitors in the 21st Century. J. Mol. Biol. 431, 3472-3500. doi: 10.1016/j.jmb.2019.04.002

Vielva, L., de Toro, M., Lanza, V. F., and de La Cruz, F. (2017). PLACNETw: a webbased tool for plasmid reconstruction from bacterial genomes. Bioinformatics 33, 3796-3798. doi: 10.1093/bioinformatics/btx462

Vinueza-Burgos, C., Ortega-Paredes, D., Narváez, C., and Zurita, J. (2019). Characterization of cefotaxime resistant Escherichia coli isolated from broiler farms in Ecuador. PLos One 14:e0207567. doi: 10.1371/journal.pone.02 07567

Wang, W., Zhao, L., Hu, Y., Dottorini, T., Fanning, S., Xu, J., et al. (2020). Epidemiological Study on Prevalence, Serovar Diversity, Multidrug Resistance, and CTX-M-Type Extended-Spectrum beta-Lactamases of Salmonella spp. from Patients with Diarrhea, Food of Animal Origin, and Pets in Several Provinces of China. Antimicrob. Agents Chemother. 64, e00092-20. doi: 10. 1128/AAC.00092-20

Yoon, E.-J., Gwon, B., Liu, C., Kim, D., Won, D., Park, S. G., et al. (2020). Beneficial Chromosomal Integration of the Genes for CTX-M Extended-Spectrum $\beta$ Lactamase in Klebsiella pneumoniae for Stable Propagation. mSystems 5, e00459-20. doi: 10.1128/mSystems.00459-20

Zankari, E., Hasman, H., Cosentino, S., Vestergaard, M., Rasmussen, S., Lund, O., et al. (2012). Identification of acquired antimicrobial resistance genes. J. Antimicrob. Chemother. 67, 2640-2644. doi: 10.1093/jac/dks261

Zhan, L., Wang, S., Guo, Y., Jin, Y., Duan, J., Hao, Z., et al. (2017). Outbreak by Hypermucoviscous Klebsiella pneumoniae ST11 Isolates with Carbapenem 
Resistance in a Tertiary Hospital in China. Front. Cell. Infect. Microbiol. 7:182. doi: 10.3389/fcimb.2017.00182

Zhang, C.-Z., Ding, X.-M., Lin, X.-L., Sun, R.-Y., Lu, Y.-W., Cai, R.-M., et al. (2019). The Emergence of Chromosomally Located blaCTX-M-55 in Salmonella From Foodborne Animals in China. Front. Microbiol. 10:1268. doi: 10.3389/fmicb. 2019.01268

Zhao, W. H., and Hu, Z. Q. (2013). Epidemiology and genetics of CTX$\mathrm{M}$ extended-spectrum $\beta$-lactamases in Gram-negative bacteria. Crit. Rev. Microbiol. 39, 79-101. doi: 10.3109/1040841X.2012.691460

Zheng, H., Zeng, Z., Chen, S., Liu, Y., Yao, Q., Deng, Y., et al. (2012). Prevalence and characterisation of CTX-M $\beta$-lactamases amongst Escherichia coli isolates from healthy food animals in China. Int J Antimicrob Agents 39, 305-310. doi: 10.1016/j.ijantimicag.2011.12.001

Zhou, Z., Alikhan, N. F., Mohamed, K., Agama Study Group, and Achtman, M. (2020). The EnteroBase user's guide, with case studies on Salmonella transmissions, Yersinia pestis phylogeny and Escherichia core genomic diversity. Genome Res. 30, 138-152. doi: 10.1101/gr.251678.119

Zurfluh, K., Albini, S., Mattmann, P., Kindle, P., Nüesch-Inderbinen, M., Stephan, R., et al. (2019). Antimicrobial resistant and extended-spectrum $\beta$-lactamase producing Escherichia coli in common wild bird species in Switzerland. Microbiolopen 8:e845. doi: 10.1002/mbo3.84

Zurita, J., Anez, F., Sevillano, G., Ortega-Paredes, D., and Paz, A. (2019). Readyto-eat street food: a potential source for dissemination of multidrug-resistant Escherichia coli epidemic clones in Quito. Ecuador. Lett. Appl. Microbiol. 70, 203-209. doi: 10.1111/lam.13263

Conflict of Interest: The authors declare that the research was conducted in the absence of any commercial or financial relationships that could be construed as a potential conflict of interest.

Copyright (c) 2021 Leão, Clemente, Moura, Seyfarth, Hansen, Hendriksen and Amaro. This is an open-access article distributed under the terms of the Creative Commons Attribution License (CC BY). The use, distribution or reproduction in other forums is permitted, provided the original author(s) and the copyright owner(s) are credited and that the original publication in this journal is cited, in accordance with accepted academic practice. No use, distribution or reproduction is permitted which does not comply with these terms. 